\title{
Las revistas y la institucionalización de la sistemática en América Latina
}

\section{Systematics' Latin American Journals and institutionalization}

\author{
Layla Michán
}

Facultad de Ciencias, Universidad Nacional Autónoma de México. Av. Universidad 3000, Circuito exterior s/n, C.U. Del. Coyoacán 04510, México, D. F., México.

Correspondencia: laylamichan@yahoo.com

\begin{abstract}
Resumen. Se realizó el análisis bibliométrico de 11185 artículos sobre sistemática de 414 revistas latinoamericanas publicadas entre 1976 y 2006 y se presentan los resultados sobre la producción general de las revistas, el país de edición y las disciplinas que incluyen. Se identifican las revistas clave, se presentan sus características y desarrollo, y se detalla la cobertura temporal, regional y temática (enfoques y taxones) de cada una. Los resultados se integran y contextualizan para identificar las características más importantes del proceso de institucionalización de la disciplina.
\end{abstract}

Palabras clave: análisis de la ciencia, bibliometría, taxonomía, base de datos Periódica.

Abstract. I undertook bibliographic analysis of 11185 systematic papers from 414 Latin American journals from 1976 to 2006. I present results from journals' general production, country of publishing, and disciplines. I identify the most productive journals, their characteristics, development, and details regarding temporal, regional and topical (focus and taxa) coverage. Results are discussed in terms of how they contributed to the institutionalization of the field.

Key words: science analysis, bibliometrics, taxonomy, Periódica database.

\section{Introducción}

El actual "análisis de la ciencia" es un área de investigación innovadora cuya finalidad es evaluar la estructura y el desarrollo de la ciencia desde una perspectiva interdisciplinaria en la que conviven la historia, la sociología, la etnología y los estudios métricos de la información. Uno de sus propósitos consiste en identificar y caracterizar cada uno de los actores y procesos involucrados en la práctica científica; a saber, los investigadores, los grupos de investigación, las instituciones, los países y la producción científica, para determinar sus relaciones y dinámica.

Los estudios métricos de la información se refieren a disciplinas emergentes, como infometría, ciencimetría, bibliometría y webometría, encargadas de estudiar los aspectos cuantitativos de la información, la ciencia, los documentos y las páginas electrónicas, respectivamente. Estos métodos fueron difundidos por Derej de Solla Price en Big science, little science (1963), y han sido impulsados de manera importante por Eugene Garfield. Los estudios bibliométricos han tenido un auge importante durante las últimas décadas, simultáneo al apogeo de las ciencias y las tecnologías de la información y comunicación, que han

Recibido: 05 marzo 2008; aceptado: 16 mayo 2008 fomentado el aumento, digitalización y sistematización de la información y la producción de una gran cantidad y diversidad de bases de datos bibliográficas. Así se manejan cantidades inmensas de datos y se aplican análisis estadísticos e indicadores matemáticos para reconocer las regularidades de la producción y la difusión de la ciencia, respecto a publicación, autoría, colaboración e impacto de las disciplinas, los temas, las regiones, los investigadores y las instituciones, por mencionar algunos. Los resultados obtenidos de los análisis métricos de la información tienen áreas de aplicación diversas; por ejemplo, los científicos los utilizan para investigar el estado de su disciplina; los historiadores, sociólogos y etnólogos de la ciencia como sustento empírico; los bibliotecarios para diseñar sus colecciones de literatura científica, y las instancias gubernamentales o institucionales, como herramienta de evaluación para el diseño de la gestión y la política científica (Albornoz, 2002). En la actualidad no existe algún trabajo infométrico sobre la sistemática mundial o de América Latina.

Tradicionalmente, los estudios bibliométricos se realizan utilizando bases de datos de corriente principal, multidisciplinarias, internacionales y de acceso restringido, especialmente el Science Citation Index Expanded (Thomson Scientific, 2008), y más recientemente Scopus 
(Elsevier, 2008). Sin embargo, estas fuentes de información no son suficientemente representativas de la literatura producida en los países en desarrollo o de idioma distinto al inglés (Gómez et al., 1999). Por ejemplo, la cifra de los artículos registrados en Periódica (Índice de revistas latinoamericanas en ciencias) es un orden de magnitud mayor que los contenidos sobre la región en cualquier otra base de datos con bibliografía científica, como Science citation index, Scopus, Pascal, CA, Biosis, CAB, Medline, Inspec y Compendex (De Moya et al., 2007; RICYT, 2007). En Ulrich's, la base de datos más extensa con información sobre publicaciones periódicas del mundo, sólo hay 4 países con más de 10 revistas: Brasil, México, Argentina y Venezuela (De Moya-Anegón et al., 2007).

Periódica (http://www.dgbiblio.unam.mx/) es una base de datos de libre acceso, disponible en línea desde 1978. En la actualidad contiene más de 270000 registros de artículos originales, informes técnicos, estudios de caso, comunicaciones cortas y otros documentos publicados en más de 1500 revistas de América Latina y del Caribe especializadas en ciencia y tecnología. Es la base de datos bibliográfica sobre ciencia latinoamericana de mayor cantidad y calidad, porque contiene el universo más completo de revistas académicas publicadas en la región, su cobertura temporal es de más de 30 años, y su cobertura temática es multidisciplinaria, amplia e incluyente (agrociencias, biología, ciencias exactas, física, geociencias, ingeniería, medicina y química). Asimismo, indiza y normaliza la información bibliográfica de cada uno de los artículos publicados y tiene ligas con algunos de los documentos en texto completo.

En la presente investigación se aplican análisis bibliométricos a las publicaciones periódicas con información sobre sistemática de América Latina editadas durante el periodo 1976-2006, para identificar las revistas que han publicado sobre el tema y reconocer los patrones de producción de cada país, así como saber cuáles son las publicaciones clave del área, descubrir sus características, y establecer el desarrollo regional y temático (enfoques y taxones) de cada una. Estos resultados permitirán identificar las características relevantes de la estructura y desarrollo de los estudios sobre biodiversidad de especies publicados en América Latina, los que se contextualizan e integran para correlacionarlos con la institucionalización de la sistemática en la región.

El proceso de institucionalización de una disciplina científica se refiere al establecimiento de instituciones en las que se realiza, desarrolla, difunde, enseña, comunica, fomenta o profesionaliza la práctica científica, como universidades, laboratorios de investigación, museos y sociedades, que en su conjunto reflejan la estructura social y el robustecimiento de una disciplina. Los productos especializados y colectivos característicos de dicho proceso son las publicaciones periódicas, uno de los indicadores más representativos de la actividad científica de una región (Vessuri, 1995).

\section{Materiales y método}

Se obtuvieron 11213 registros de artículos sobre sistemática de la base de datos Periódica a cargo de la Dirección General de Bibliotecas-UNAM (DGB-UNAM) con los que se creó una sub-base "Periódica-taxonomíasistemática". Este proceso constó de la selección y recuperación de los registros en una base y la migración hacia la otra, implicando en cada caso un proceso cíclico que constó de varias normalizaciones, depuraciones, revisiones, validaciones y pruebas, tanto unitarias, como globales (de tendencias generales), para asegurar la fiabilidad y robustez de la información.

Se identificaron y seleccionaron (24 de enero del 2007) todos los artículos que estuvieran clasificados en el campo "Tema" con la categoría "taxonomía y sistemática". La asignación de los campos así como la captura de toda la información de Periódica está realizada por especialistas, con base en la lectura completa del texto del artículo y la indización de los términos se hace utilizando lenguaje poscoordinado, es decir, el analista asienta cada palabra por separado. Esto representa una ventaja para la investigación bibliométrica, pues se hace discriminación e interpretación crítica de la información contenida en cada documento (no lo hace una máquina con criterios masivos), lo que repercute en la definición correcta de la muestra, el uso de los términos idóneos, y asegura la homogeneidad y consistencia en la información registrada. Los criterios de inclusión de revistas en Periódica se pueden consultar en DGB (2008).

Para cada artículo se recuperaron hasta 13 campos con información sobre 3 elementos: 1), el documento: tipo, formato, enfoque (hasta 3), tema (hasta 3), título, idioma, idioma del resumen, palabras clave y keywords (hasta 8), volumen, número, mes(es), parte, páginas; 2), la revista: título, país, año, ISSN, y 3), los autores: nombre del autor (hasta 8), institución y país. Los detalles al respecto se pueden consultar en Michán et al. (2008). Las revistas clave se identificaron aplicando el modelo matemático de Bradford (Gorbea, 1996). Para realizar este análisis se identificaron los títulos que corresponden a unidades históricas, es decir, las revistas que han cambiado de nombre o publican series dedicadas a distintas especialidades, resultado de la evolución de las propias disciplinas y del proceso de institucionalización. 


\section{Resultados}

Después de la normalización y depuración de la información se identificaron $11 \quad 185$ artículos y 411 revistas sobre sistemática en un periodo de 30 años (1976 a 2006), que constituyeron el $23.15 \%$ de los artículos, el $25.9 \%$ de las revistas y el $71.4 \%$ de los países contenidos en Periódica. El promedio de publicación de artículos fue de 372 por año, la mediana 404, el máximo fue de 503 en 1993 y el mínimo 17 en 1976.

\section{Discusión}

En la figura 1 se presenta el comparativo de la publicación de revistas y artículos en el tiempo. En la medida que crece el interés sobre una especialidad o disciplina determinada, asciende su producción científica, y por consiguiente, aumenta la dispersión de los artículos especializados que se publican (Gorbea, 2005). El crecimiento neto de la cantidad de artículos sobre sistemática fue escaso, sobre todo si se considera la cantidad de especies que falta por describir, resultado consistente con la idea de que la sistemática está viviendo un periodo crítico caracterizado por la falta de recursos económicos y humanos (Soberón y Llorente, 1993; Godfray, 2002; 2007;
Wheeler et al., 2004; Michán et al., 2008). Esta debilidad se extiende a América Latina, pero incrementada por la fragilidad institucional de la ciencia en la región.

Las primeras publicaciones periódicas "científicas" de América datan del último tercio del siglo XVIII, desde esa época hasta ahora la publicación de revistas ha sido un proceso lleno de dificultades, similar al de las sociedades e instituciones que las editaron, su existencia ha sido alterada por los continuos problemas políticos, económicos y sociales. Fue hasta mediados del siglo XX que varias de las instituciones científicas del área se consolidaron (Vessuri, 1996), simultáneamente a sus medios de difusión, las revistas. De acuerdo con Periódica, la revista con temas taxonómicos más antigua, todavía vigente, es la Revista del Museo de La Plata que tiene sus antecedentes en 1864, cambió de nombres y perdura hasta ahora. Se pueden ver detalles en el portal de la institución editora: la Facultad de Ciencias Naturales y Museo de La Plata, República Argentina (2008).

Para 1978, en Periódica había cerca de una decena de revistas; en 1981 alcanzó la cifra de 89 y de este año a 2005 la mediana fue de 78 por año (Fig. 1). El número máximo de revistas con temas taxonómicos ingresadas en Periódica por año fue de 91, en 1982; por lo tanto, el resto, 325 (78\%), no fue capturado continuamente. Estas variaciones se deben a diversas razones; por ejemplo, para que las

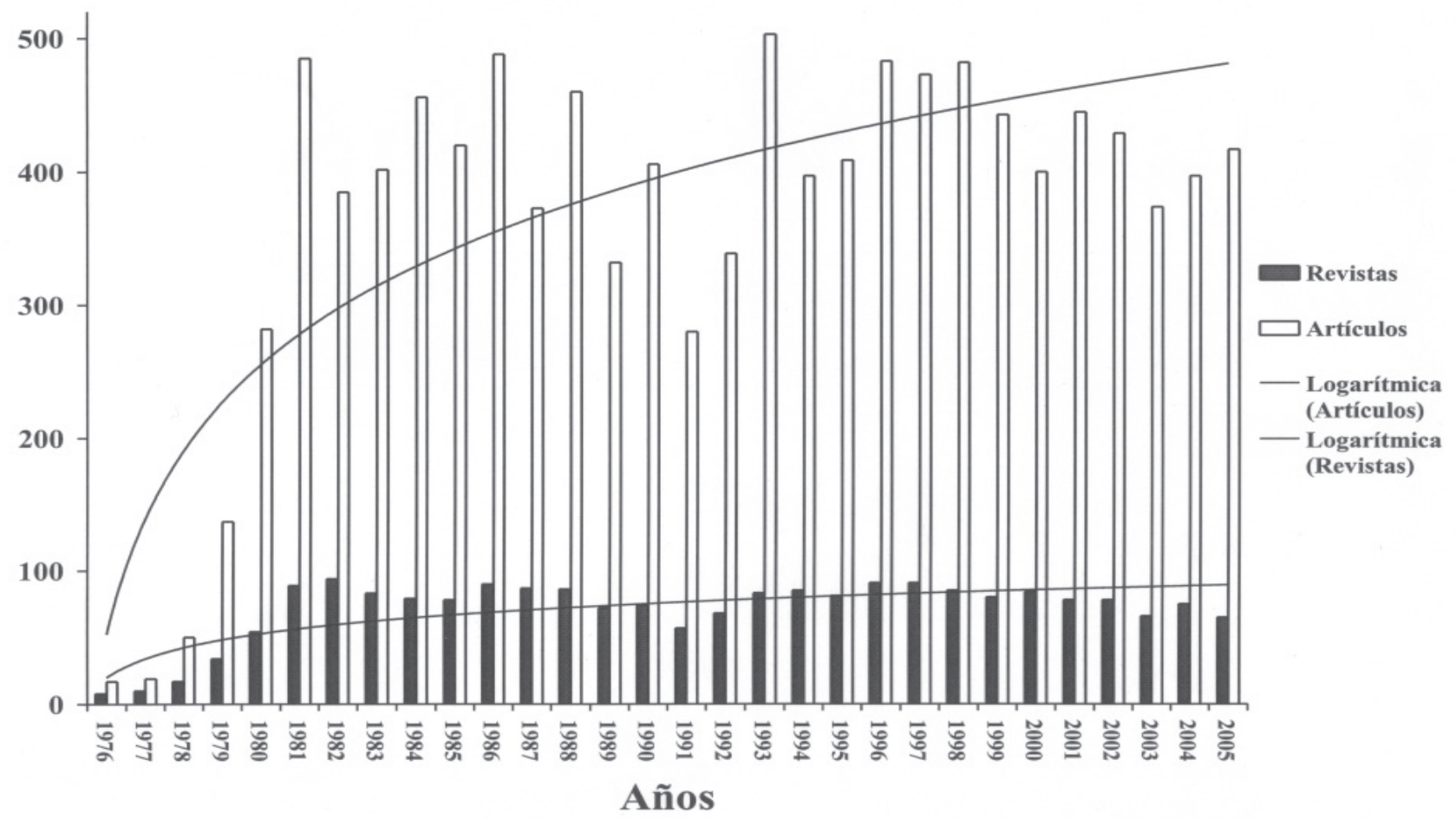

Figura 1. Tendencia temporal de los artículos y las revistas de la sub-base taxonomía-sistemática de Periódica. 
revistas fueran capturadas era necesario tener el ejemplar impreso físicamente, en muchos casos esto no fue posible, ya fuera por retraso en su publicación, por lejanía, por lentitud del correo, porque el editor no la envía o porque falta personal suficiente para dar seguimiento preciso a cada una de ellas. Esto se ha subsanado actualmente cuando hay disponible versión electrónica en texto completo de acceso libre a tales revistas. Otra razón es que varias tienen una publicación irregular. Generalmente la publicación de sus números se atrasa debido a falta de recursos, carencia de artículos originales de calidad y lentitud en los procesos de revisión y edición, e incluso muchas de ellas se han dejado de publicar, entre otros problemas comunes de las publicaciones periódicas latinoamericanas en la actualidad (Laufer, 2007a-c).

En la figura 2 se puede apreciar la cantidad de primeros números publicados, de las 204 revistas que tienen mayor cantidad de artículos en Periódica. Esta gráfica representa la tendencia de aparición de nuevas revistas. Se ve un aumento significativo en los años 60 y 70, periodo que coincide con la formación de un gran número de universidades, institutos de investigación y sociedades en la región (Vessuri, 1996).

Se identificó un total de 411 títulos de revistas sobre sistemática editadas en 15 países de América Latina (ninguna estuvo clasificada como internacional, una es estadounidense), resaltaron Brasil, México (ambos clasificados como megadiversos) y Argentina, las cuales contienen el $72.4 \%$ de los artículos y el $68.6 \%$ de los autores que abordaron temas taxonómicos (Fig. 3). Respecto a la proporción de artículos publicados por cada región y los que tratan sobre cada país fue evidente que, en general, los países publican principalmente los estudios realizados en su propia región; pero Colombia, por ejemplo, publica más en revistas extranjeras que propias, pues tiene en proporción pocas revistas sobre el tema (Fig. 3).

De acuerdo con los análisis bibliométricos realizados sobre la ciencia latinoamericana, la producción científica de Brasil es casi el doble de la mexicana (Cetto y Vessuri, 1998; Cetto y Vessuri, 2005; Palatano, 2005; SCImago, 2008). En Periódica están sobrerrepresentadas, como es normal, las revistas producidas en el país de origen de la base de datos (México) (Alonso Gamboa y Reyna Espinosa, 2005). La subrepresentación de las brasileñas se debe a la lejanía y al idioma. Los resultados de esta investigación han sido aplicados para normalizar la representación de las revistas y se han añadido buena cantidad de revistas brasileñas a Periódica para subsanar este problema.

Del resto de los países megadiversos de la región, Colombia, Venezuela y Perú tuvieron una escasa contribución de revistas. Chile y Cuba aparecieron en cuarta y tercera posición. La información respecto a la isla

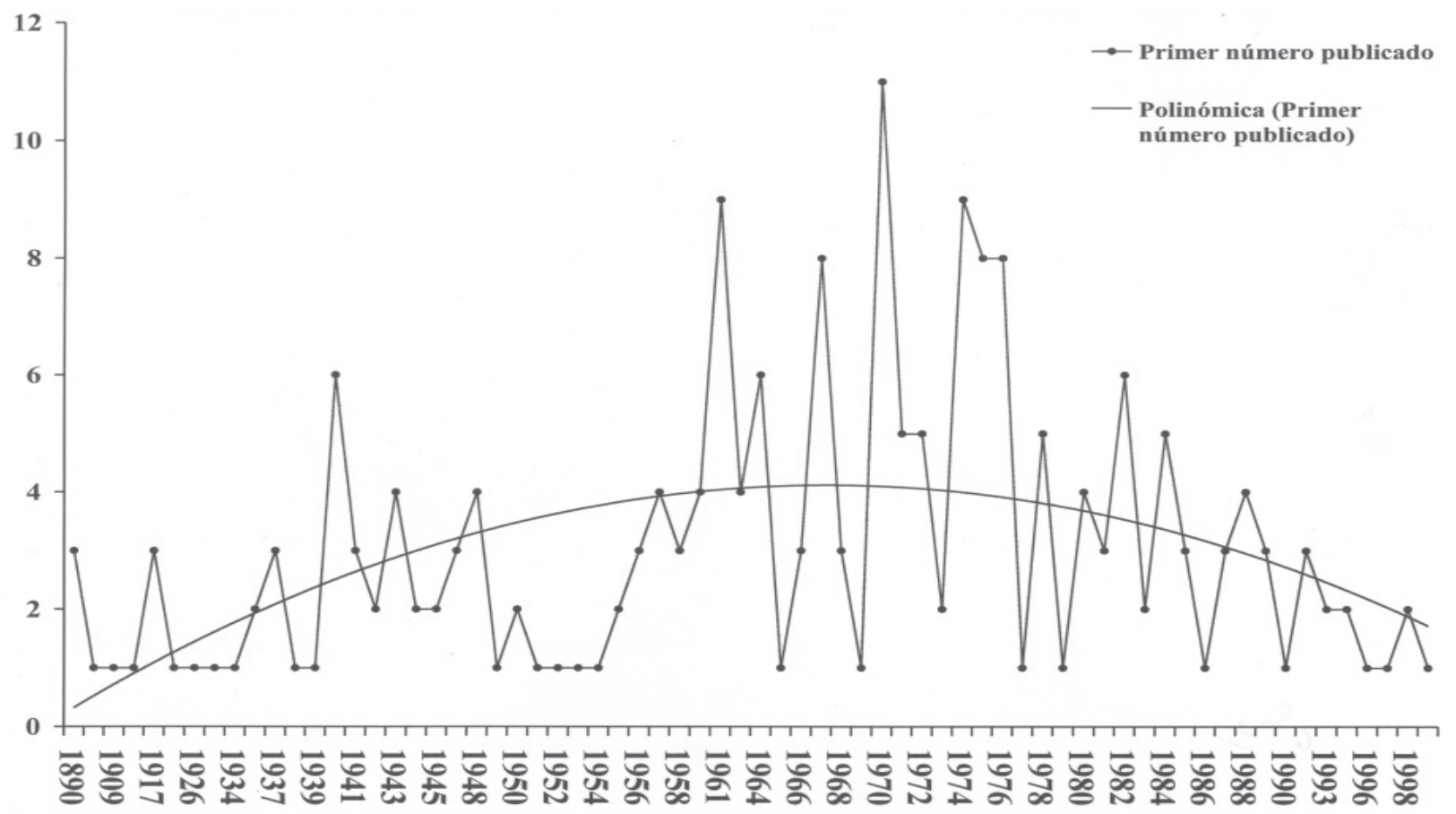

Figura 2. Tendencia temporal de la publicación del primer número de las revistas en la sub-base taxonomía-sistemática de Periódica. 


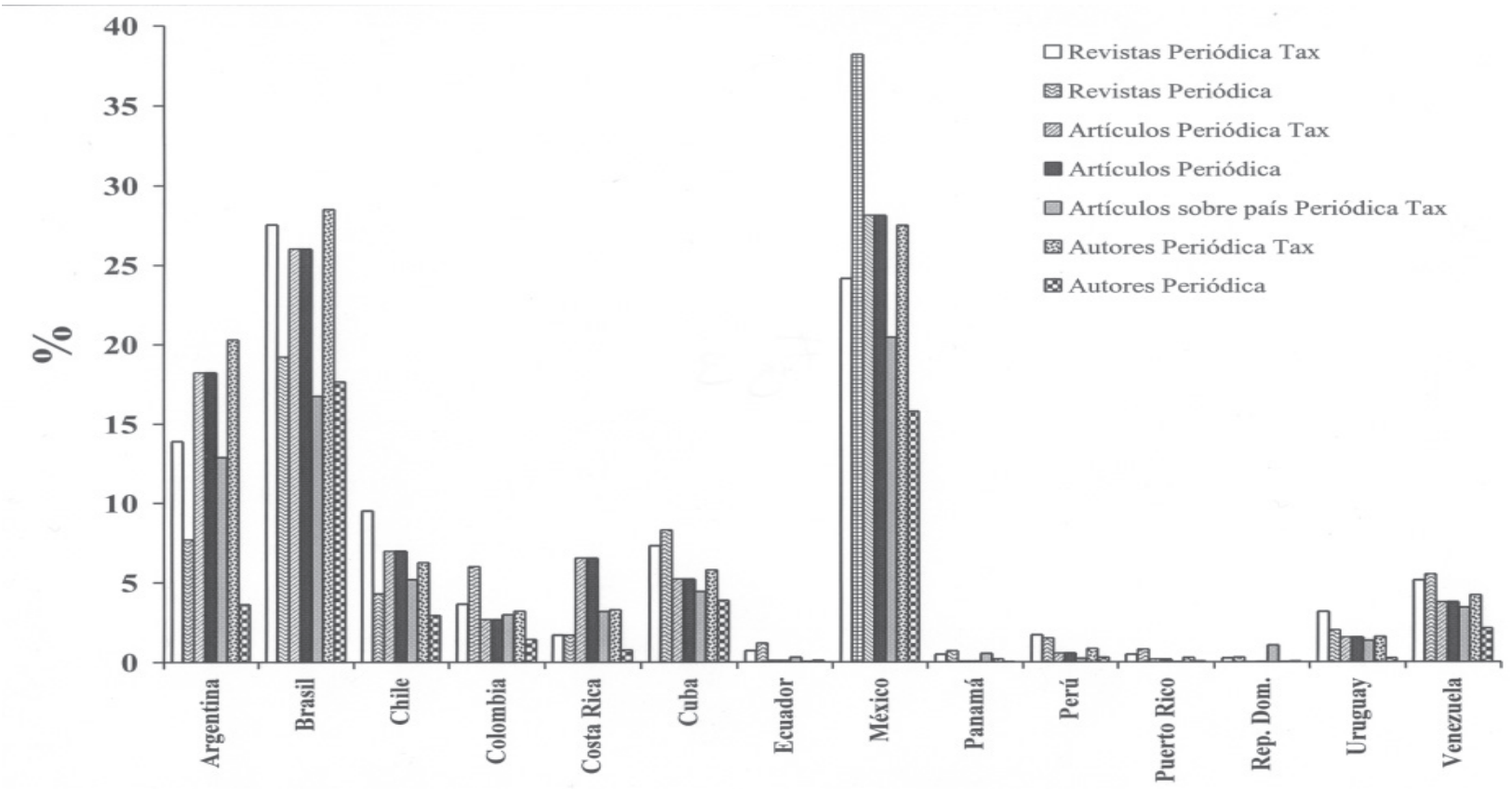

Figura 3. Revistas, artículos y autores publicados en Periódica y en la sub-base taxonomía-sistemática. Las revistas y los artículos de Periódica se obtuvieron de Alonso Gamboa y Reyna Espinosa (2005).

es trascendente, ya que su producción no está registrada en las bases de datos internacionales. Guatemala, El Salvador, Nicaragua y Honduras carecieron de revistas sobre taxonomía, aunque cuentan con revistas de otras disciplinas registradas en Periódica (Fig. 3).

Las revistas clave. De los 414 títulos de revistas identificadas, 371 correspondieron a unidades históricas (véase Material y método). A falta de otros criterios más completos, las revistas más significativas fueron elegidas usando únicamente la productividad, ya que Periódica carece de información sobre referencias y citas. Se obtuvo la primera zona de producción con 10 títulos y $3823(34.18 \%)$ artículos, una segunda de 27 títulos y 3 $611(32.28 \%)$ artículos, y una tercera de 335 títulos con 3751 (33.54\%) artículos. Los resultados obtenidos y el comportamiento de la curva no se ajustaron al modelo matemático de Bradford (Bradford, 1948; Gorbea, 1996). Se identificaron los 37 títulos de revistas más productivas o núcleo (zonas 1 y 2 sensu Bradford), que representan el $9.97 \%$ del total de títulos registrados sobre sistemática y corresponden a 7434 (66,8\%) de los artículos (Fig. 4). Algunos detalles bibliográficos sobre cada revista clave se presentan en el Cuadro 1.

En cuanto al formato de los documentos publicados por las revistas clave, el $86 \%$ fueron artículos y el resto, notas breves o noticias, monografías, catálogos, reseñas de libro, resúmenes, revisiones bibliográficas, conferencias o discursos, biografías, informes técnicos, ensayos, planes, proyectos, programas, editoriales y cronologías.

Respecto al idioma, naturalmente éste estuvo relacionado con el país de origen; 25 revistas clave (47\%) publicaron artículos sólo en español, 32 (60\%) admitieron artículos en español, 12 (22.6\%) en portugués, 10 (18.8\%) en inglés y 15 (28.3\%) son revistas que publican artículos en más de un idioma (inglés, español, portugués y en algunos casos francés, alemán e italiano), resultado que refleja claramente la diversidad y riqueza lingüística (Cuadro 1).

En relación con la cantidad de artículos de cada unidad histórica (Fig. 4), la que aportó mayor número fue la recientemente renombrada Revista Mexicana de Biodiversidad, fundada en 1930 y editada por el Instituto de Biología, UNAM. Anteriormente se denominó Anales del Instituto de Biología de la Universidad Nacional Autónoma de México, series Botánica (hasta 2005), Zoología (hasta 2005), Biología Experimental (hasta 1980) y Ciencias del Mar y Limnología (hasta 1974), nombres que reflejaban las diferentes líneas de investigación que se practicaban en la institución. Años después, paralelo a la evolución de institución, su órgano de difusión experimentó una renovación, se fusionó, cambio de nombre, formato y la estructura del comité editorial (León Règagnon, 2005). Lo mismo sucedió en 1999 con la Revista del Museo Argentino de Ciencias Naturales (Nueva serie). Estos 


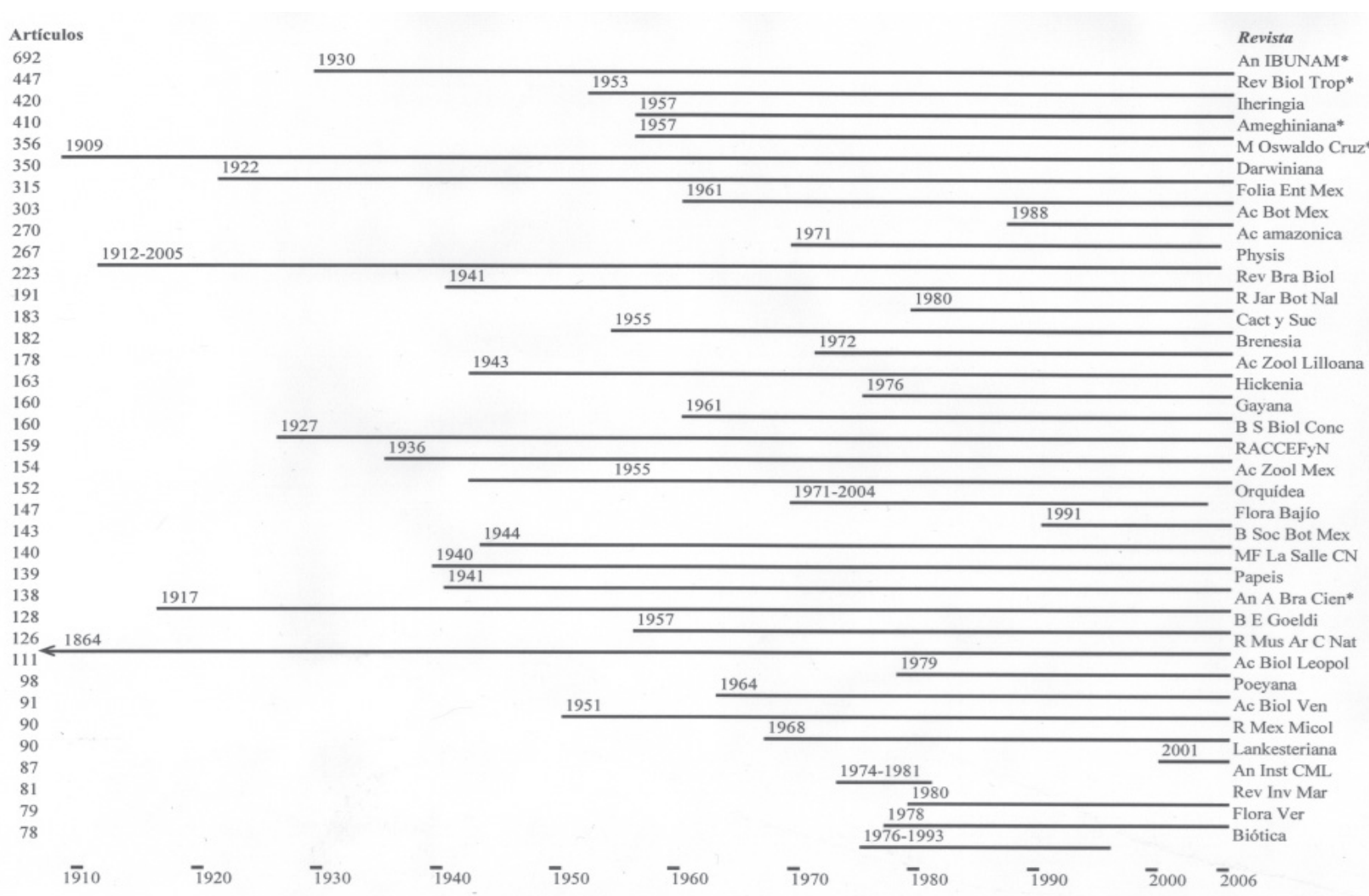

Figura 4. Cobertura temporal, fecha de fundación y la cantidad de artículos registrados para cada revista clave (unidades históricas). Las revistas con asterisco (*) están indizadas en el SCI. El título correspondiente a cada abreviatura se puede consultar en el Cuadro 1.

cambios representan claramente la correlación existente entre el desarrollo y la estructura de las disciplinas, las instituciones y la producción generada.

Una manera de evaluar la calidad de las revistas es a través de la indización en los sistemas de información especializados, como el Science citation index (SCI) (Palatano, 2005; Aguirre et al., 2006). La producción de las revistas clave estuvo directamente relacionada con el impacto, 5 de ésas están indizadas en el SCI, por lo que se consideran de corriente principal y prestigio internacional, ocupan las posiciones de productividad 1, 2, 4, 5 y 26, respectivamente (Fig. 4). Además, 43 revistas clave están registradas en Biosis $(81 \%)$ y $34(64 \%)$ aparecen en Zoological record.

La cobertura temporal de las revistas clave se representa también en la figura 4, donde se especifica la fecha en que apareció el primer número de cada unidad histórica, que se denomina con el título más representativo (Cuadro 1). Sólo una revista fue fundada en el siglo XIX, el resto aparecieron durante el XX, por lo tanto, las revistas locales sobre taxonomía son jóvenes, comparadas con aquellas europeas fundadas en el siglo XVII, como Philosophical Transactions y Biological Journal of the Linnean Society que perduran hasta nuestros días. Lankesteriana sólo lleva 6 años publicándose y ya está colocada dentro de las revistas más productivas.

El análisis de la periodicidad de las revistas clave mostró resultados interesantes. Diez títulos tienen aparición irregular, el resto la tienen bimestral (3), trimestral (8), cuatrimestral (5), semestral (14) y anual (3) (Cuadro 1), de manera que $74 \%$ de ellas tiene una periodicidad mayor a 3 meses, lo que es mucho tiempo si se considera la velocidad con la que se produce nuevo conocimiento en el área.

De las 37 unidades históricas $4(11 \%)$ se han dejado de publicar: Physis (perduró 93 años), Orquídea (22 años), Anales del Instituto de Ciencias del Mar y Limnología (29 años) y Biotica (12 años) (Cuadro 1, Fig. 4). Sobre la primera de ellas explícitamente sabemos que fue debido a la carencia de fondos (Scioscia, 2005).

Un hito importante en la historia de las revistas científicas fue su publicación electrónica, lo que ha disminuido costos y aumentado enormemente la difusión 


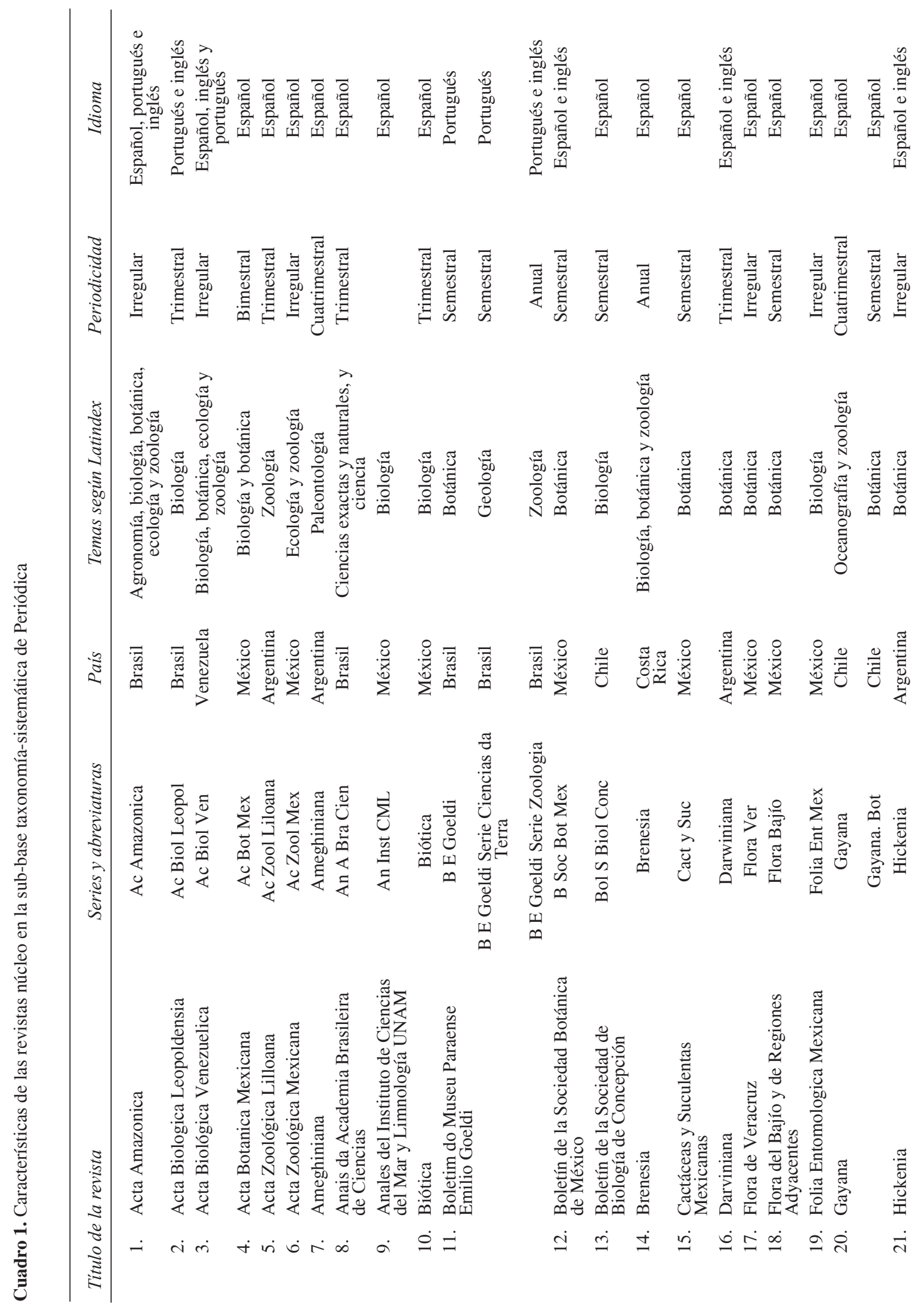




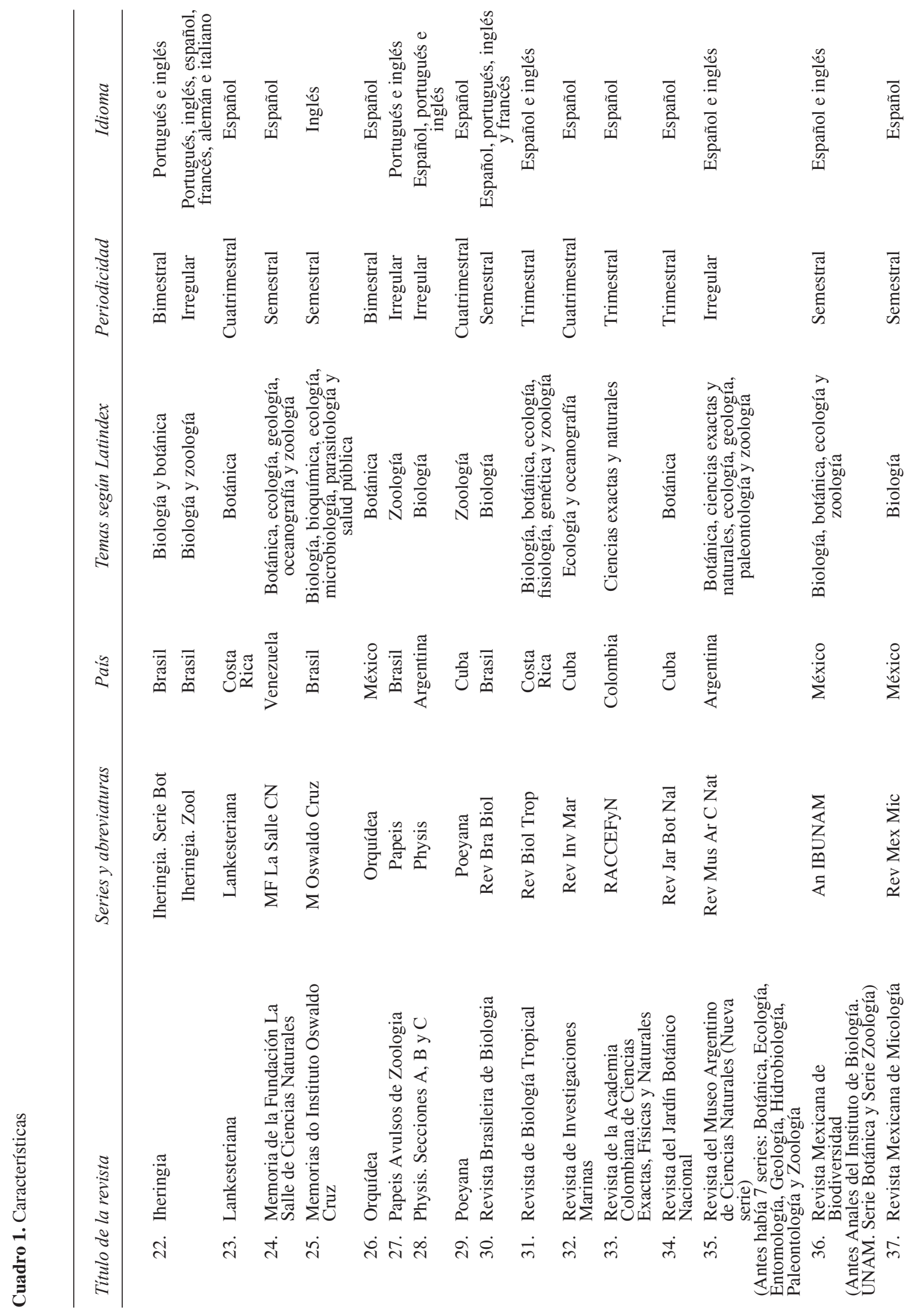


de sus contenidos. Acorde a esta tendencia, todas las revistas vigentes tienen al menos una página electrónica en línea y la mayoría de ellas están disponibles en texto completo con acceso libre.

Respecto a las instituciones editoras de las revistas clave, el $66 \%$ fueron producidas por entidades académicas: institutos de investigación (28\%), universidades (13\%) $\mathrm{y}$ museos $(25 \%)$, las cuales cuentan generalmente con subvención gubernamental; $32 \%$ fueron de sociedades, academias y fundaciones; las particulares tuvieron sólo un $2 \%$. Estas cifras coinciden con las obtenidas para las bases de datos Clase y Periódica (Alonso Gamboa y Reyna Espinosa, 2005) y para la taxonomía mexicana (Michán y Llorente, 2003).

De acuerdo con la clasificación de Latindex (2007), la base de datos con información bibliográfica más completa sobre las revistas latinoamericanas, $19(35.8 \%)$ tratan sobre botánica, 15 (28.3\%) sobre biología, 11 (20.7\%) sobre zoología, $4(7.5 \%)$ sobre paleontología o geología y 4 (7.5\%) son de ciencia en general (Cuadro 1). De tal forma que los artículos taxonómicos se publicaron en las revistas sobre biología en general (en varios casos denominada historia natural) o que tratan sobre grupos taxonómicos (animales, plantas y microorganismos) o subdivisiones taxonómicas de ellos.

Ninguna revista de América Latina tiene en el título la palabra taxonomía o sistemática, tampoco hay alguna dedicada exclusivamente a la taxonomía. Pero sólo hay un puñado de revistas en el mundo que tratan sobre el tema de manera casi exclusiva y que abarcan cualquier taxón (v. gr. Cladistics y Systematic Biology).

Estos resultados son característicos de la sistemática, una práctica científica no unificada o apenas en unificación, que practican diversos profesionales de la biología, de acuerdo con su objeto de estudio (zoólogos, botánicos y microbiólogos), que provienen de tradiciones distintas (Nordenskiöld, 1949). Todos ellos utilizan técnicas, métodos, conceptos, jerarquías e incluso hasta códigos de nomenclatura distintos, entre otras cosas; lo que los relaciona son sus objetivos (nombrar, describir, clasificar, establecer relaciones evolutivas, etcétera).

El análisis del campo "tema" (se asignan hasta 3 temas para cada documento) permitió conocer a detalle los contenidos publicados por cada unidad histórica. El 100\% de los artículos versaron sobre sistemática (puesto que estaban clasificados con ese tema), pero sus contenidos se concentraron en 10 temas relacionados, ya fueran disciplinas o taxones estudiados. Los grupos más frecuentes fueron los insectos y las angiospermas, abordados por $22(60 \%)$ y $21(56.7 \%)$ revistas, respectivamente. La categoría "zoología" no aparece en el cuadro porque a los animales generalmente se les asigna como tema el grupo taxonómico al que corresponden (Cuadro 2).

En cuanto a la concentración geográfica obtenida del campo "palabra clave", 5487 artículos (49\% del total) tuvieron asignado 1 o varios países: 150 artículos nombraron 2, 23 refirieron 3, y 3 apuntaron 5 y 6 países, respectivamente. Gran parte de los artículos (5308) mencionaron un solo país, y es generalmente el mismo en el que se edita la publicación periódica. Hay 36 revistas clave que contienen entre el $75 \%$ y el $100 \%$ de artículos que tratan sobre su propio país, de acuerdo con ese criterio serían locales (Fig. 5). La Revista de Biología Tropical publicó menos del $50 \%$ de artículos sobre su país de origen, de tal forma que sería la de mayor cobertura regional y de acuerdo con el SCI (Thomson Scientific, 2008) también es la que tiene mayor índice de impacto.

La correlación existente entre el contenido geográfico de los documentos y el lugar o país de publicación de éstos, o de los autores, fuentes o instituciones que los generan, puede considerarse como una medida del interés o desinterés que un país o región puede manifestar sobre su propia problemática o entorno nacional, o sobre la perteneciente a otros países y regiones (Gorbea, 2005). Este fenómeno se da en la sistemática, los principales interesados en conocer lo publicado sobre biodiversidad de especies de América Latina son los lectores locales y regionales, ya que los recursos bióticos representan prioridades nacionales.

El desarrollo de las revistas que publicaron sobre taxonomía tiene una relación directa con la institucionalización de la disciplina. Para que cada una de esas revistas se originara, produjera y difundiera fue necesaria la existencia de una estructura institucional, conceptual, social e incluso económica. La institucionalización de la taxonomía latinoamericana, así como la historia de sus publicaciones periódicas, ha sido un proceso tardío, lento y frágil. Es evidente la juventud y la inestabilidad de las revistas, es notoria la falta de infraestructura y la carencia de recursos. Pero también es evidente, pese a las condiciones adversas, el gran esfuerzo que han hecho los especialistas de la región por consolidar sus disciplinas y producir medios especializados para la discusión y difusión de sus ideas.

Toda la información existente produce una rica diversidad que forma la infodiversidad, la cual representa pluralidad, rescate, conservación, disponibilidad, libre acceso a la información y es la defensa de las múltiples y distintas manifestaciones de la generación y creación de la información y, a su vez, la conservación de las ideas y los pensamientos que han existido (Morales, 2003). Es imperativo reconocer la importancia cultural, científica y económica que representan las publicaciones periódicas latinoamericanas para el desarrollo nacional y regional; y 


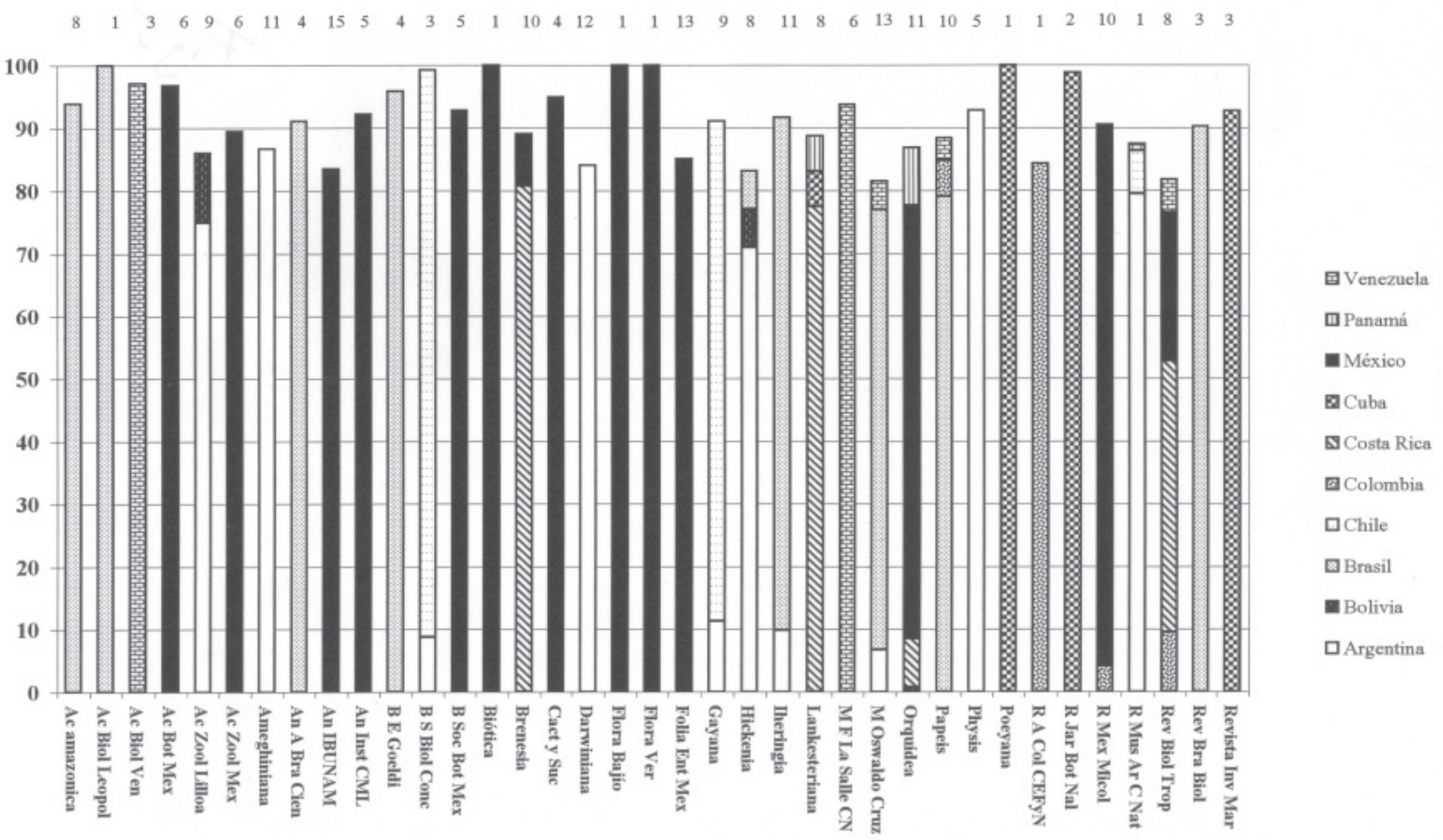

Figura 5. Países en los que se realizaron los estudios publicados en cada revista. Los números en la parte superior se refieren a la cantidad total de países abordados para cada revista.Se grafican los países con representación de $10 \%$ o más.

fortalecerlas, utilizarlas y citarlas (Ríos Gómez y Herrero Solana, 2005), en especial las que presentan estudios sobre biodiversidad, por ser un tema estratégico tanto regional como local.

Los resultados expuestos en este documento son representativos de la producción local y regional sobre sistemática en América Latina, no sólo porque se obtuvieron de la fuente con mayor cobertura temática, temporal y regional, sino porque los patrones de publicación de los científicos de los países periféricos, como es el caso de los latinoamericanos, se dividen en 2 grupos, los pocos que publican en las publicaciones periódicas de corriente principal y la mayoría que publican en revistas nacionales y regionales (Russell, 1998: Russell et al., 2007). Sin embargo, representan una aproximación parcial, pues sólo se consideraron las revistas y la literatura endógena (publicada en las revistas latinoamericanas). Para tener una visión completa respecto a la estructura y desarrollo de los estudios sobre la biodiversidad de latinoamericana en la actualidad, es necesario también considerar otros elementos relacionados con el proceso de institucionalización, los artículos y los investigadores. En cuanto a los primeros, quien esto escribe ha hecho un estudio sobre su producción, tipo de estudios y trabajos, los grupos taxonómicos, los temas, el formato y el idioma (Michán et al., 2008); en cuanto a los segundos, también está en preparación un trabajo sobre la autoría, la coautoría y la colaboración, con base en información de Periódica. Pero no sólo eso, es indispensable incluir también la producción exógena (publicada en revistas extranjeras), tarea que la autora realiza con el análisis de información de bases de datos como Biosis, Science Citation Index Expanded, CAB, Zoological Record y PubMed, que se publicará en breve.

Entre 1976 y 2006 se publicaron más de 414 revistas latinoamericanas, editadas en 15 países, que publicaron 11 125 artículos. La aparición de nuevas revistas fue mayor en las décadas de 1960 y 1970, el máximo número de revistas que se han registrado para un año fue de 91 en 1982.

Se identificaron $37(10 \%)$ revistas clave, que produjeron más del $65 \%$ de los artículos sobre sistemática de la región, éstas trataron sobre botánica (36\%), biología (28\%), zoología (20\%), paleontología o geología (7.5\%) y ciencia en general (7.5\%). Los grupos y los enfoques más frecuentes fueron los insectos y las angiospermas.

Las revistas más productivas (zona 1, sensu Bradford) fueron en orden descendiente: Revista Mexicana de Biodiversidad, Revista de Biología Tropical, Ameghiniana, 
Cuadro 2. Porcentaje de los principales temas que aborda cada revista de acuerdo con el campo "tema" de la sub-base taxonomíasistemática de Periódica. El título correspondiente a cada abreviatura se puede consultar en el Cuadro 1

\begin{tabular}{|c|c|c|c|c|c|c|c|c|c|c|c|c|}
\hline ڤ్ & $\begin{array}{l}\text { Uू } \\
\stackrel{\Xi}{\Xi} \\
\text { s. }\end{array}$ & 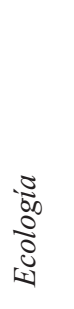 & 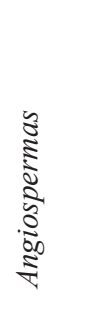 & 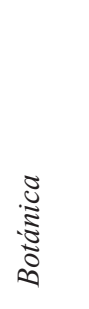 & 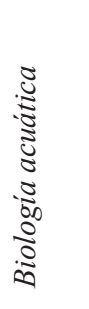 & 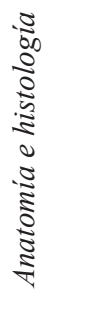 & 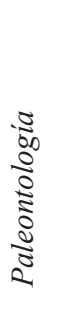 & 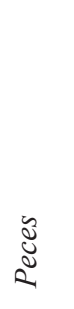 & 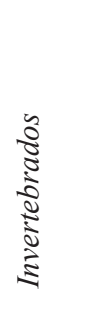 & 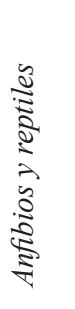 & 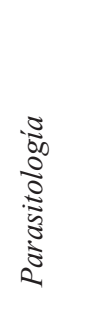 & $\begin{array}{l}\text { Dे } \\
0 \\
\tilde{\Xi} \\
\vdots\end{array}$ \\
\hline Ac Amazonica & 38 & 26 & 16 & 16 & 10 & 1 & & 6 & 3 & & 6 & 4 \\
\hline Ac Biol Leopol & 13 & 40 & 5 & 1 & 13 & 15 & & 21 & 4 & 20 & & 1 \\
\hline Ac Biol Ven & 14 & 19 & 4 & 6 & 17 & 13 & & 28 & 4 & 11 & 10 & \\
\hline Ac Bot Mex & & 15 & 69 & 16 & 2 & 14 & & & & & & 11 \\
\hline Ac Zool Liloana & 53 & 21 & 1 & & 11 & 10 & 1 & 3 & 2 & 20 & 5 & \\
\hline Ac Zool Mex & 67 & 21 & & 1 & 1 & 6 & & & 4 & 6 & 3 & \\
\hline Ameghiniana & & 6 & 5 & 15 & 6 & 5 & 99 & & 11 & 11 & & 1 \\
\hline An A Bra Cien & 25 & 6 & 6 & 6 & 10 & 21 & 50 & 13 & 8 & 10 & & \\
\hline An IBUNAM & 48 & 27 & 10 & 7 & 10 & 7 & & 5 & 3 & 2 & 10 & 2 \\
\hline An Inst CML & 1 & 58 & 1 & & 80 & 8 & & 23 & 23 & & 4 & \\
\hline B E Goeldi & 32 & 24 & 23 & 2 & 2 & 15 & 3 & 2 & 2 & 10 & 1 & 2 \\
\hline B S Biol Conc & 32 & 28 & 3 & 1 & 27 & 19 & 2 & 13 & 14 & 3 & 6 & 1 \\
\hline B Soc Bot Mex & 0 & 32 & 28 & 50 & 6 & 6 & & & & & & 2 \\
\hline Biótica & 1 & 32 & 46 & 31 & 5 & 26 & 4 & 1 & & & & 8 \\
\hline Brenesia & 18 & 37 & 26 & 12 & 10 & 4 & 1 & 2 & 6 & 2 & 3 & 10 \\
\hline Cact y Suc & 1 & 22 & 84 & 19 & & 7 & & & & & 1 & \\
\hline Darwiniana & & 16 & 59 & 16 & 5 & 7 & & & & & & 9 \\
\hline Flora Bajío & & 22 & 66 & 31 & 1 & 4 & 1 & & & & & \\
\hline Flora Ver & & 18 & 67 & 32 & & & & & & & & \\
\hline Folia Ent Mex & 93 & 28 & & & 5 & 6 & & & & & 2 & 1 \\
\hline Gayana & 34 & 26 & 13 & 6 & 14 & 8 & 1 & 9 & 5 & 2 & 4 & \\
\hline Hickenia & 1 & 3 & 56 & 27 & & & & & & & 1 & 3 \\
\hline Iheringia & 41 & 13 & 10 & 1 & 8 & 9 & & 3 & 8 & 1 & 2 & 1 \\
\hline Lankesteriana & & 1 & 76 & 8 & & 1 & & & & & & 1 \\
\hline MF La Salle CN & 7 & 46 & 6 & 11 & 17 & 14 & 1 & 15 & 4 & 17 & 1 & \\
\hline M Oswaldo Cruz & 50 & 8 & & & 11 & 10 & 1 & 7 & 1 & 1 & 29 & \\
\hline Orquídea & 1 & 9 & 83 & 38 & & 8 & & & & & & \\
\hline Papeis & 45 & 14 & & & 2 & 11 & 3 & 7 & 6 & 28 & 3 & \\
\hline Physis & 22 & 20 & 1 & 5 & 36 & 11 & & 3 & 10 & 2 & 3 & 3 \\
\hline Poeyana & 16 & 27 & & & 12 & 15 & 5 & 1 & 11 & 11 & 7 & \\
\hline RACCEFyN & 8 & 6 & 30 & 15 & 1 & 10 & & 2 & & 23 & 1 & 4 \\
\hline R Jar Bot Nal & 1 & 20 & 48 & 19 & 2 & 14 & & & & & 1 & 20 \\
\hline R Mex Micol & 2 & 25 & 1 & 1 & & 3 & & & & & 1 & 98 \\
\hline R Mus Ar C Nat & 18 & 17 & 3 & 14 & 14 & 11 & 20 & 10 & 6 & 4 & 1 & 1 \\
\hline Rev Biol Trop & 18 & 54 & 4 & 8 & 19 & 7 & 1 & 14 & 13 & 8 & 3 & 4 \\
\hline Rev Bra Biol & 39 & 13 & 6 & 6 & 7 & 8 & & 5 & 8 & 6 & 7 & \\
\hline Revista Inv Mar & & 35 & 1 & & 49 & 10 & & 14 & 16 & & & \\
\hline
\end{tabular}


Memorias do Instituto Oswaldo Cruz, Darwiniana, Folia Entomologica Mexicana, Iheringia, Acta Botanica Mexicana, Acta Amazonica y Physis.

Una proporción importante de revistas y artículos fue publicada por México, Brasil y la Argentina. Únicamente 5 revistas tienen impacto internacional. El 96\% de los artículos que hicieron referencia a algún país mencionaron sólo uno y coincide con el país de origen de la revista, todas excepto la Revista de Biología Tropical fueron clasificadas como locales.

Las revistas estudiadas contienen principalmente información local y regional estratégica y significativa sobre biodiversidad en español, portugués e inglés. Su componente territorial es decisivo, de suerte que un porcentaje importante de la investigación difundida adquiere su plena relevancia en función de las características y necesidades nacionales.

La institucionalización de la sistemática en la región ha sido heterogénea y difícil. La consolidación de las publicaciones periódicas en la actualidad es producto del esfuerzo de las comunidades científicas de la región. Es importante propiciar la consolidación de las revistas existentes, publicar los resultados interesantes en revistas regionales y en el idioma propio, fomentar entre los científicos latinoamericanos el leer, utilizar y citar más los trabajos de los colegas de la región, lo que favorecerá que aumente su impacto y la permanencia de la infodiversidad de la zona. Es imperativo también desarrollar, mantener e integrar las colecciones bibliográficas de datos (bases de datos) sobre biodiversidad regional, que contengan información bibliográfica endógena exhaustiva, consistente y sistematizada sobre los taxones de la región, ya que a diferencia de otras áreas biológicas, la literatura taxonómica no caduca, y cualquier dato publicado sobre alguna especie es de mucho valor. En esta era de la información, en la que se han desarrollado gran cantidad de herramientas digitales que permiten contar con los datos necesarios, de manera eficaz, fácil e inmediata, aprovecharlos representa ya no un lujo, sino una necesidad para disminuir la brecha entre la ciencia de corriente principal y la periférica.

\section{Agradecimientos}

Los resultados de este artículo de investigación son parte del proyecto de investigación posdoctoral "Análisis del desarrollo de los estudios sobre biodiversidad en América Latina en la actualidad", en el que colaboran Antonio Sánchez Pereyra y Antonia Llorens Cruset del Departamento de Bibliografía Latinoamericana, Dirección General de Bibliotecas-UNAM, quienes no sólo han contribuido con la extracción de la información de la sub-base Taxonomía-sistemática de Periódica, sino con su experiencia. A Alejandra Diabb, por la ayuda en la realización del Cuadro 1 y la figura 3. A Salvador Gorbea Portal, por la plantilla para la realización del cálculo de Bradford. A Juan J. Morrone y 2 revisores anónimos, quienes con sus sugerencias me ayudaron a realizar la versión final de este artículo. A Óscar Flores Villela, Antonio Sánchez Pereyra, Antonia Llorens Cruset y Judith Aguirre, por la revisión del manuscrito. A Carlos López Beltrán, Jorge Llorente Bousquets, Jane Russell, Estela Morales, Armando Luis, Blanca Hernández e Isabel Vargas, por todo el apoyo que me brindaron para la realización de mi investigación. Este trabajo se realizó gracias al apoyo del programa PROFIP, DGAPA, UNAM.

\section{Literatura citada}

Aguirre, M., A. M. Cetto, S. Córdoba, Flores, A. María y A. Román. 2006. Calidad editorial y visibilidad de las revistas. La experiencia de Latindex. In Edición electrónica, bibliotecas virtuales y portales para las ciencias sociales en América Latina y El Caribe, D. Babini y J. Fraga (comps.). CLACSO (Consejo Latinoamericano de Ciencias Sociales), Buenos Aires. p. 103-122.

Albornoz, M. 2002. Situación de la ciencia y la tecnología en las Américas. Centro de Estudios sobre Ciencia, desarrollo y educación superior, Buenos Aires. 52 p.

Alonso Gamboa, J. O. y F. R. Reyna Espinosa. 2005. Compilación de datos bibliométricos regionales usando las bases de datos Clase y Periódica. Revista Interamericana de Bibliotecología 28:63-78.

Bradford, S. C. 1948. Documentation. Crosby Lockwood, London. $196 \mathrm{p}$.

Cetto, A. M. y H. Vessuri. 1998. Latin America and the Caribbean, world science report 1998. In UNESCO science report. UNESCO, Paris. p. 55-75.

Cetto, A. M. y H. Vessuri. 2005. Latin America an the Spanishspeaking Caribbean science report 2005. In Science report 2005. UNESCO, París. p. 45-76.

Elsevier B.V. 2008. Scopus. http://www.scopus.com/scopus/ home.url Consultada: 16 febrero 2008

De Moya-Anegón, F., Z. Chinchilla-Rodríguez, B. VargasQuesada, E. Corera-Álvarez, F. J. Muñoz-Fernández, A. González-Molina y V. Herrero-Solana. 2007. Coverage analysis of Scopus: a journal metric approach. Scientometrics 73:53-78.

DGB-UNAM (Dirección General de Bibliotecas-UNAM). 2008. Criterios de selección de revistas para las bases de datos Clase y Periódica, Dirección General de Bibliotecas, UNAM, México, D. F. http://132.248.9.1:8991/F/4XJNCCG5D3 9F9IXS8QXXE8BIXJVJAADK9HNV6FLCD55I84QXE 1-00529? func $=$ file \&file_name $=$ crit-sel-per01; consultada: 16.I.2008.

Facultad de Ciencias Naturales y Museo, República Argentina. 2008. Relanzamiento de la Revista del Museo de La Plata. 
http://www.fcnym.unlp.edu.ar/publi/revista/index_abajo. html; consultada: 18.I.2008.

Godfray, H. C. J. 2002. Challenges for taxonomy - The discipline will have to reinvent itself if it is to survive and flourish. Nature 417:17-19.

Godfray, H. C. J., B. R. Clark, I. J. Kitching, S. J. Mayo y M. J. Scoble. 2007. The web and the structure of taxonomy. Systematic Biology 56:943-955.

Gómez, I., R. Sancho, L. Moreno y M. T. Fernández (1999) Influence of Latin American journals coverage by international databases. Scientometrics 46:443-456.

Gorbea, S. 1996. El modelo matemático de Bradford: Su aplicación a las revistas latinoamericanas de las ciencias bibliotecológica y de la información. Centro Universitario de Investigaciones Bibliotecológicas, UNAM, México, D. F. $152 \mathrm{p}$.

Gorbea, S. 2005. Modelo teórico para el estudio métrico de la información documental. Trea, Gijón. 171 p.

Latindex. 2007. Latindex. Sistema regional de información en línea para revistas científicas de América Latina, el Caribe, España y Portugal. http://www.latindex.unam.mx/latindex/ busquedas1/latin.html; consultada: 13 noviembre 2007.

Laufer, M. 2007 a, b, c. La cultura de la publicación científica (1), (2) y (3). Interciencia 32:501, 577 y 653.

León Règagnon, V. 2005. Presentación. Revista Mexicana de Biodiversidad 76:1-2.

Michán, L. y J. Llorente. 2003. La taxonomía en México durante el siglo XX. Publicaciones Especiales del Museo de Zoología, Facultad de Ciencias, UNAM 1:1-250.

Michán, L., J. Russell, A. Sánchez Pereyra, A. Llorens Cruset y C. López Beltrán 2008. Análisis de la sistemática actual en Latinoamérica. Interciencia 33:754-761.

Morales, E. 2003. Infodiversidad, globalización y derecho a la información. Sociedad de Investigaciones Bibliotecológicas, Buenos Aires. 203 p.

Nordenskiöld, E. 1949. Evolución histórica de las ciencias biológicas. Espasa-Calpe, Buenos Aires. 715 p.

Palatano, M. 2005. Las publicaciones del campo científico: las revistas académicas de América Latina. Anales de
Documentación 8:217-235.

Price, D. S. 1963. Big science, little science. Columbia University, New York. 119 p.

RICYT. 2007. Red de Indicadores de Ciencia y TecnologíaIberoamericana e Interamericana: Indicadores comparativos. http://www.ricyt.edu.ar/interior/interior.asp?Nivel1=1\&Niv el2=2\&Idioma=; consultada: 5.X.2007

Ríos Gómez, C. y V. Herrero Solana. 2005. La producción científica latinoamericana y la ciencia mundial: una revisión bibliográfica (1989-2003). Revista Interamericana de Bibliotecología 28:43-61.

Russell, J. M. 1998. Publishing patterns of Mexican scientists: Differences between national and international papers. Scientometrics 41:113-124.

Russell, J. M., J. A. Del Río y H. D. Cortés. 2007. Highly visible science: A look at tree decades of research from Argentina, Brazil, Mexico and Spain. Interciencia 32:629-634.

SCImago. 2008. SCImago Journal \& Country Rank. http://www. scimagojr.com/; consultada: 19.II.2008.

Scioscia, C. L. 2005. Mensaje Revista Physis escrito por la tesorera de la Asociación Argentina de Ciencias Naturales y editora responsable de la Revista Physis. http://www. bioetica.org/physis.htm; consultada: 8.II.2008.

Simpson, G. G. 1961. Principles of Animal Taxonomy. Columbia University Press, New York. 247 p.

Soberón, J. y J. Llorente. 1993. The use of species accumulation functions for the prediction of species richness. Conservation Biology 7:480-488.

Thomson Scientific. 2008. Science citation index (SCI). http:// scientific.thomson.com/products/sci/; consultada: 16 febrero 2008.

Vessuri, H. 1995. Recent strategies for adding value to scientific journals in Latin America. Scientometrics 34:139-161.

Vessuri, H. 1996. La ciencia académica en América Latina en el siglo XX. In Historia social de las ciencias en América Latina, J. J. Saldaña (comp.). Universidad Nacional Autónoma de México/Porrúa, México, D. F. p. 437-479.

Wheeler, Q. D., P. H. Raven y E. O. Wilson. 2004. Taxonomy: impediment or expedient? Science 303:285-285. 